\title{
SELF-HEALING CAPACITY OF CONCRETE - COMPUTER SIMULATION STUDY OF UNHYDRATED CEMENT STRUCTURE
}

\author{
Huan He, Zhandi Guo, Piet Stroeven, Martijn Stroeven And \\ LAMBERTUS JOHANNES SLUYS
}

Faculty of Civil Engineering and Geosciences, Delft University of Technology, Stevinweg 1, 2628CN Delft, The Netherlands

e-mail: H.He@tudelft.nl

(Accepted October 26, 2007)

\begin{abstract}
Aggregate occupies at least three-quarters of the volume of concrete, so its impact on concrete's properties is large. The aggregate's influence on the non-hydrated part of the matured paste is assessed by concurrent algorithm-based computer simulation system SPACE in this paper. A distinction is made between interfacial zones (ITZs) and bulk paste. Containers with rigid boundaries were employed for the production of series of cement pastes. They were subjected to quantitative microstructure analysis. Relevant gradient structures in the ITZ and bulk are presented and discussed. The relevance of this structure information for possible selfhealing of cracks is briefly discussed.
\end{abstract}

Keywords: aggregate, computer simulation, self-healing, unhydrated cement nuclei.

\section{INTRODUCTION}

Concrete is a composite material consisting basically of aggregate and cement paste. Different from the concept of a continuous "matrix" in which aggregate particles are embedded, as assumed in many studies (Pigeon, 1969; Lusche, 1972; Farahat et al., 1995), the aggregate forms a skeleton structure requiring all particles to be included in the simulation. The average nearest neighbor aggregate grain surfaceto-surface spacing in normal concrete is of the order of $50 \mu \mathrm{m}$ (Scrivener et al., 2004), in a range of $0.1 \sim 200 \mu \mathrm{m}$ (Chen et al., 2005). Most of the cement paste is therefore pocketed between two or more aggregate grains, whereby a major portion will consist of interfacial zones (ITZs) with properties deviating from those in bulk. Density is lower, and porosity and its connected fraction are found concentrated inside these ITZs (Chen et al., 2005; 2006).

Shrinkage and local secondary displacements during maturation lead to the formation of myriads of tiny cracks in the concrete's cement paste, the major part of which will be situated inside the ITZs (Stroeven, 1973). Additional microcracks can be initiated, of course, by freeze-thawing, temperature gradients and mechanical loading. The dispersion of such cracks is inevitably governed by the dispersion of the aggregate grains in the jammed state. These fine cracks can heal under favorable moisture conditions, to which Turner (1973) referred as autogenous healing. Several mechanisms of self-healing are proposed (Neville, 1995), i.e., hydration of the hitherto unhydrated cement, formation of insoluble calcium carbonate, and mechanical blocking by very fine material suspended in the water.

This paper will focus in a computer simulation approach on the first mechanism, whereby the selfhealing capability can be expected primarily depending on the amount of unhydrated cement. However, also number density and spacing distribution of unhydrated cement nuclei (UCN) could play a role, defining it as a configuration-sensitive phenomenon (Stroeven, 1973). This paper will discuss the "realistic" simulation of the spatial UCN structure by the Delft-produced concurrent algorithm-based SPACE system (Stroeven and Stroeven, 1997; Stroeven, 1999). Experimental information seems limited to self-healing effects in concrete elements provided with a single small crack subjected to mechanical loading (Kasperkiewicz and Stroeven, 1991; Granger et al., 2005). Further, only scare experimental evidence is available as to technological influences (such as w/c ratio and cement fineness) on the self-healing capacity of concrete (Liu et al., 2005). 


\section{MATERIALS AND METHODOLOGY}

Three different types of Portland cement (PC) are considered in this study with w/c ratios of $0.2,0.3$, $0.4,0.5$, and 0.6. The PC types are denoted by C206, C319, and C497; i.e., C206 is cement with Blaine number 206 reflecting its specific surface area in $\mathrm{m}^{2} / \mathrm{kg}$. The respective grain size distribution functions comply with the Rosin-Rammler size distribution function (van Breugel, 1997; Stroeven, 1999; Hu, 2004)

$$
G(d)=1-\exp \left(-b d^{a}\right),
$$

in which $a$ and $b$ are constants, to be specified for each cement mix. Fig. 1 shows the cumulative size distribution curves of the model cements, with grain sizes ranging from 1 34.72, 1 26.15 and 1 18.84 $\mu \mathrm{m}$ for C206, C319 and C497, respectively.

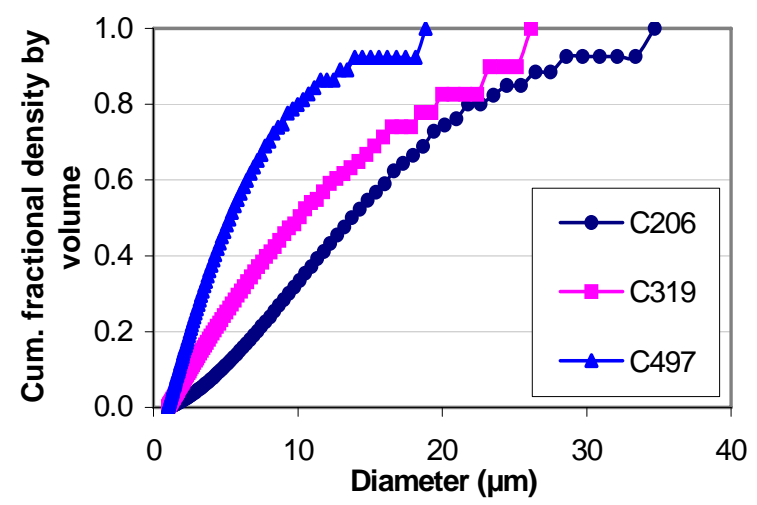

Fig. 1. Cumulative particle size distribution (PSD) curves for simulated cements.

Mixtures of 5000 cement particles conforming to the three PSDs of Fig. 1 were considered in the simulation. Final volume fractions of $35 \%$, 39\%, $44 \%, 51 \%$ and $61 \%$ correspond to w/c ratios of 0.6 , $05,0.4,0.3$, and 0.2, respectively. Each parameter combination was represented in this preliminary study by a single cube. The final linear dimensions of the cubes were in the range 75.7 91.1, 51.3 61.7 and 40.7 49 $\mu \mathrm{m}$ for C206, C319 and C497, respectively. Hence cube size is smaller than the RVE (or RAE) for even composition. However, the size ratios of cubes and RVEs for composition (about four times maximum grain size) are quite similar all over the testing range, as shown in Table 1 . When it is assumed that the RVE/RAE size for a given configurationsensitive descriptor is a certain (descriptor-sensitive!) factor exceeding the one for composition homogeneity, an unbiased comparison study would still be possible.
At least observed trends should be considered reliable, therefore.

Table 1. Ratios of linear dimensions of cubes and RVE/RAEs for composition.

\begin{tabular}{|l|ccccc|}
\hline \multicolumn{1}{|c|}{ Cement w/c } & 0.2 & 0.3 & 0.4 & 0.5 & 0.6 \\
\hline C206 & 0.55 & 0.58 & 0.61 & 0.63 & 0.66 \\
C319 & 0.49 & 0.52 & 0.55 & 0.57 & 0.59 \\
C497 & 0.54 & 0.57 & 0.60 & 0.63 & 0.65 \\
\hline
\end{tabular}

The discrete element simulation system SPACE models the fresh paste as a set of spherical elements dispersed in water. Since the elements represent real physical phases in the material, physical properties can be assigned to each element along with its size. There are two vital stages incorporated in the SPACE system (Stroeven, 1999). A dilute 3-D distribution of elements with predefined size distribution is generated in the initial stage in a container. Next, random linear and rotational velocity vectors are assigned to each element. Then in the dynamic stage, the location and orientation of all particles are changed at each time step according to a Newtonian motion model, while the container size is reduced. Finally, the iteration stops when a certain condition is met, e.g., the relevant volume fraction of particles is reached. Another vital stage is the hydration process. Individual hydrating cement particles are represented by sets of concentric spheres in the early stages of the hydration process. The kinetics of the hydration process is governed in an initial stage by a boundary mechanism, followed by one in which the reaction rate is controlled by a diffusion mechanism. For more detailed information, see Stroeven (1999) and Stroeven and Stroeven (1999). The hydration algorithms largely correspond to those of van Breugel (1997).

\section{RESULTS}

\section{GLOBAL FEATURES OF UCN STRUCTURE}

Fig. 2 shows a selection of the cements and their one-year-old SPACE-generated structures of hydrated cements and of UCN for various technological parameters. This type of information offers a visual perception on the UCN structures as functions of these technological parameters. Next step is the geometrical statistical (i.e., stereological) description of aspects of these structures, so that a comparative study can be conducted. 


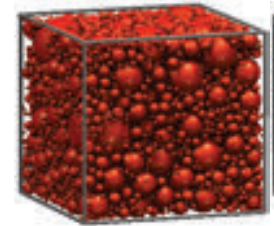

$\mathrm{w} / \mathrm{c}=0.2$

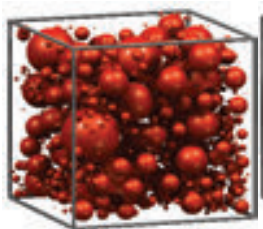

$\mathrm{w} / \mathrm{c}=0.2$

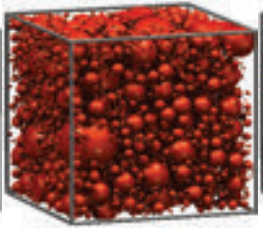

$\mathrm{w} / \mathrm{c}=0.4$

$\mathrm{w} / \mathrm{c}=0.6$

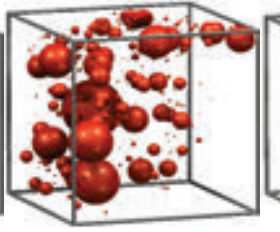

$\mathrm{w} / \mathrm{c}=0.4$ $\mathrm{w} / \mathrm{c}=0.6$

Fig. 2. Cement pastes (top) and UCN (bottom) of C206 after 1 year of hydration.

The simplest one is the configuration-insensitive volume fraction descriptor, $V_{V}$. Data in Fig. 3, pertaining to the average value of volume density in the cube, demonstrate the fineness of the cement to have only a marginal effect on this parameter at equal w/c ratio. The many tiny unhydrated cement nuclei only marginally influence this parameter. However, this is different for the average number density in the cube, or the number of nuclei per unit of sample volume, $N_{V}$. The sensitivity level was set at $0.01 \mu \mathrm{m}$; smaller nuclei are not expected exerting significant effects on the self-healing capacity. Results are presented in Fig. 4.

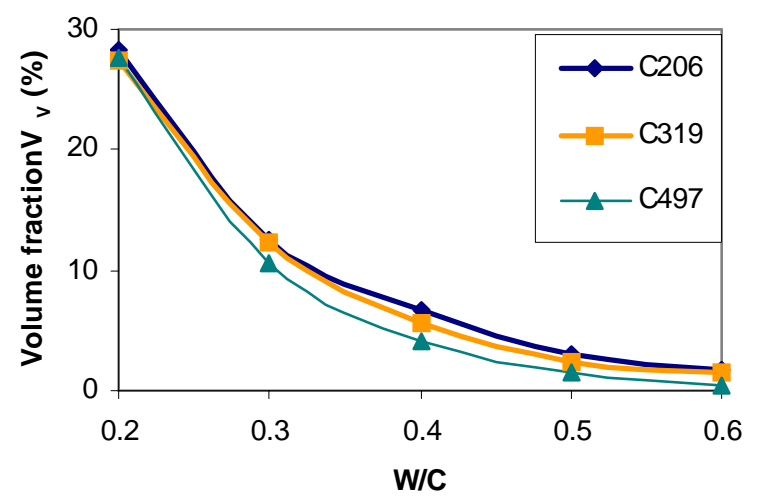

Fig. 3. Volume fraction of UCN for three cements with different $w / c$ ratios.

The same sensitivity level was maintained for the highly configuration-sensitive nearest neighbor surfaceto-surface spacing (NNSSS). A single example is presented in Fig. 5 for w/c $=0.2$. Larger cubes would have resulted in smoother curves. Smoothening can also be achieved by averaging over a series of identical tests.

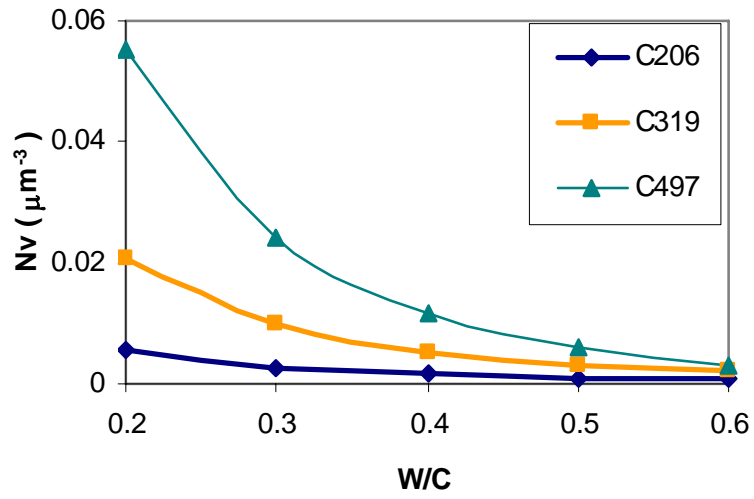

Fig. 4. Number of UCN per unit volume for three cements with different $w / c$ ratios.

The different measures for the curve's characteristic averages are approximately increasing linearly with water to cement ratio, as depicted by Fig. 6 . Hence, cement more completely hydrates at higher w/c ratio and the resulting smaller numbers of nuclei left unhydrated are as a consequence more remote from each other.

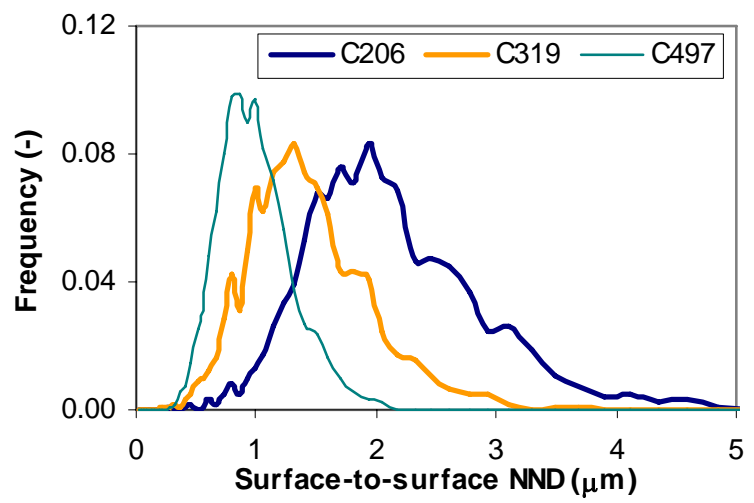

Fig. 5. Probability density functions of NNSSS of UCN in three cement pastes $(w / c=0.2)$.

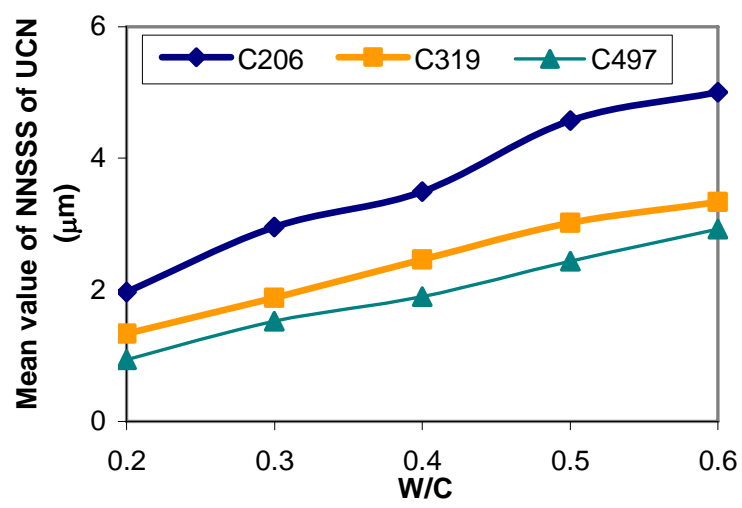

Fig. 6. Mean values of NNSSS-curves of three cements paste with increasing $w / c$ ratios. 


\section{LOCAL FEATURES OF UCN STRUCTURE}

So far, the ITZ properties are globally characterized. They can be compared with global bulk values. Gradients in local values perpendicular to the aggregate grain's surface have been determined for UCN, gel and porosity. Fig. 7 only shows as an example $V_{V}$-gradients of UCN in C497.

Fig. 7 and other gradient structures of UCN, gel and porosity clearly reveal the wall effects in $V_{V}$, defining the extent of the associated ITZ. Order of magnitude of the ITZ's extent for volume fraction is $10 \mu \mathrm{m}$ for the applied cements defined in Fig. 1 (slightly increasing with w/c ratio, as shown earlier in Stroeven (1999; 2005). The fractional volume density of UCN declines from bulk toward the aggregate surface. This is more pronounced the lower the water to cement ratio. Although not demonstrated because of space limitations, an opposite conclusion can be drawn with respect to porosity. Fig. 8 presents the same data however comparing the different cement types for the sole case of w/c $=0.2$ (since Fig. 3 demonstrated the effect of fineness on fractional volume density to be marginal). The slope in the gradient structures is the highest for the fine-grained cement, although leading to roughly the same level in bulk volume density as was earlier shown in Fig. 3. Hence, low w/c-ratio and high cement fineness basically promote arriving at higher volume densities in the proximity of the aggregate grain surfaces. This will reduce probability of crack development in this region.

Figs. 9 and 10 indicate surface area density to be influenced by both technological parameters. Figs. 9 and 10 demonstrate that surface area density in ITZs is larger than in bulk for only the lowest w/c ratio case (0.2), so in the HPC range. A reversed tendency is found at higher $w / c$ ratios $(\geq 0.4)$.

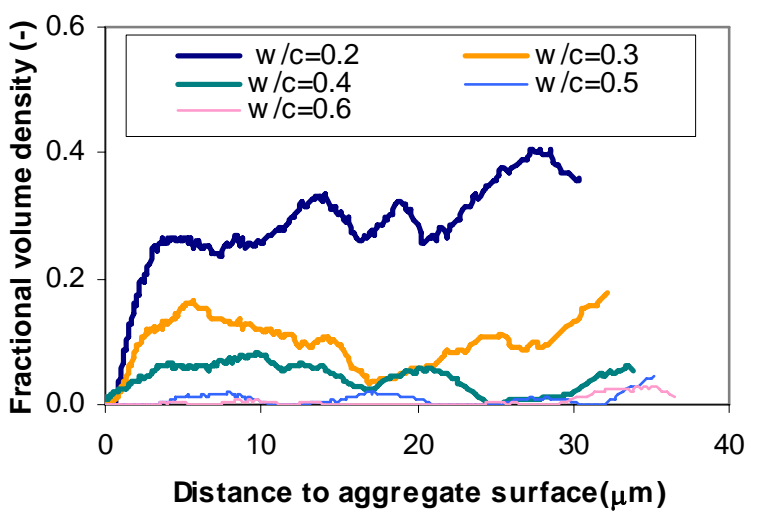

Fig. 7. Gradients in $V_{V}$ of $U C N$ in $C 497$ for five $w / c$ ratios.

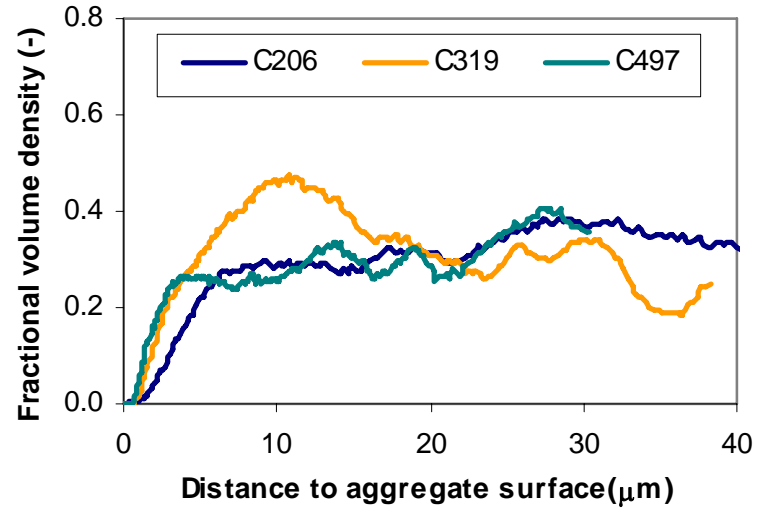

Fig. 8. Gradient in $V_{V}$ of $U C N$ for three cement paste finenesses $(w / c=0.2)$.

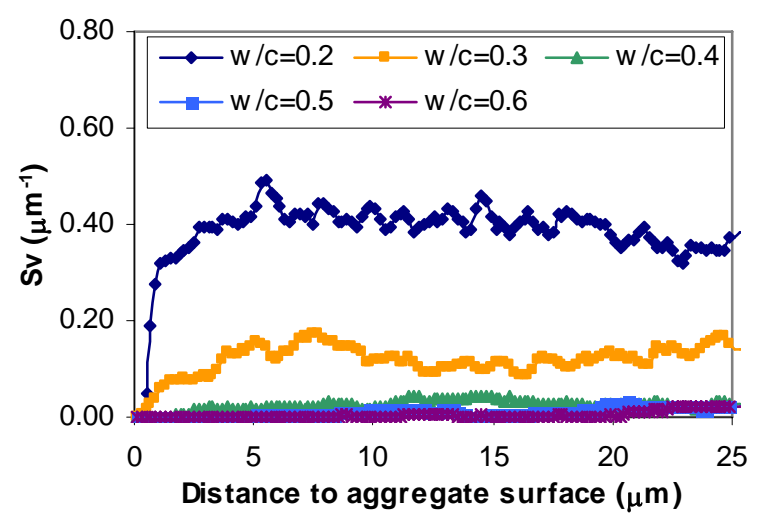

Fig. 9. Gradients in $S_{V}$ of UCN of five $w / c$ ratios (C497).

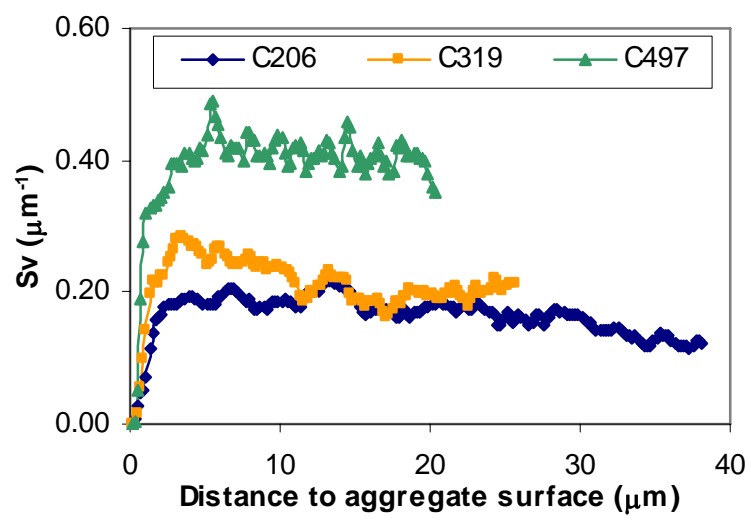

Fig. 10. Gradients in $S_{V}$ of UCN for three cement finenesses $(w / c=0.2)$.

The mean free spacing $\lambda$, defined as the mean uninterrupted surface-to-surface distance between all neighboring particles, is another relevant 3-D structure parameter. It can be calculated for the UCN structure by

$$
\lambda=4 \frac{1-V_{V}}{S_{V}},
$$


and as a consequence reveals the dependence of surface area density on the technological parameters, as demonstrated in Fig. 11 for global values; the finer cement specimen has somewhat lower mean free spacing of UCN $(\mathrm{w} / \mathrm{c} \leq 0.4)$. The local gradient structure information is presented in Fig. 12. Though the mean free spacing of UCN is high close to the aggregate grain's surface (reflecting high porosity level), it rapidly diminishes to its plateau value at a distance of about $5 \mu \mathrm{m}$. The ITZ thickness seems smallest for the finest cement.

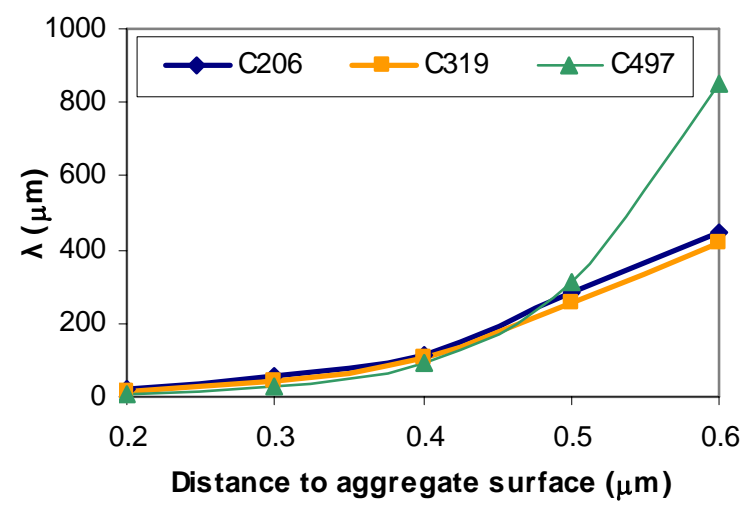

Fig. 11. Mean free spacing $\lambda$ for different types of cement and different w/c-ratios.

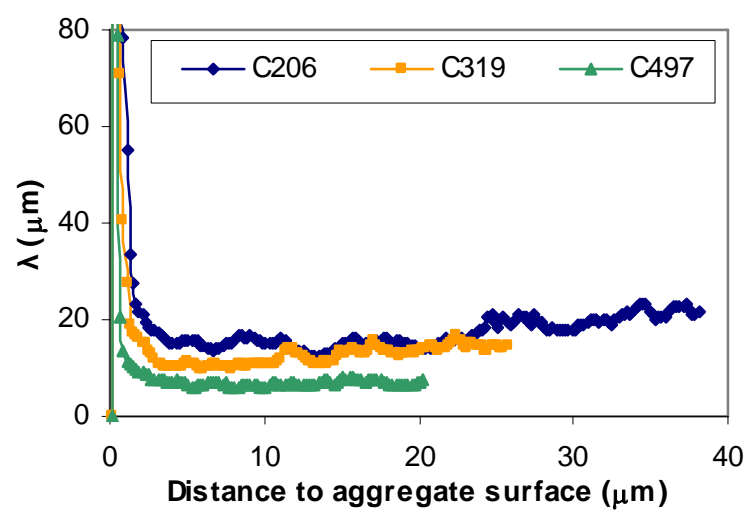

Fig. 12. Gradients in mean free spacing $\lambda$ for different types of cement $(w / c=0.2)$.

\section{DISCUSSION}

The influences are investigated in this paper of water cement ratio and cement fineness on the structure of UCN that is underlying the self-healing capacity of concrete.

Volume fractions of UCN are almost similar at equal w/c ratio, and decline at higher w/c ratios as depicted by Fig. 3. Volume fraction is becoming insignificant for $\mathrm{w} / \mathrm{c} \geq 0.4$, in agreement with Power's model (Jensen and Hansen, 2001). Obviously, the associated high water contents allow for more complete hydration, and as a consequence smaller amounts of UCN will be left. Water cement ratio is compared to cement fineness the dominating factor for the selfhealing capacity of concrete, as additionally shown by Fig. 3. At higher w/c ratio, in bulk and in ITZ alike, averages of NNSSS of UCN increase; nuclei are more widely spread.

The cement fineness level exerts significant impact on the number and surface area densities of UCN $\left(N_{V}\right.$, $S_{V}$ ) within a limited range of water to cement ratios, i.e., 0.2 to 0.4, as illustrated in Figs. 4 and 10. In this range, the finest cement (C497) reveals much higher number and surface area densities, which can be attributed to the initially large value of $N_{V}$ in the fresh state of the cement. On the other hand, the finer cement leads to lower averages of NNSSS of UCN despite the degree of hydration (DOH) being only slightly different.

Similar global results as above mentioned were also found in simulations without ITZs (i.e., in bulk). This is not presented here because of space limitations. But for local configuration-sensitive information of cement paste, influence of aggregate is significant. A relatively high volume fraction of UCN and apparent high surface area density were found in ITZs of finer cement model paste versus coarser one (in the range of $0 \sim 5 \mu \mathrm{m}$ in Fig. 8).

The ITZ is most liable to crack formation (Stroeven, 1973; Jacobsen et al., 1995). The probability of microcracks in these zones to interfere with the UCN can be expected depending at least on the volume fraction of UCN. Furthermore, a tendency of cracks to follow the external surface of the UCN was found experimentally (Baldie, 1985, Struble et al., 1989). Baldie explains this phenomenon by conceiving the UCNs as strong inclusions in the hardened cement paste. Therefore, surface area density, $S_{v}$, of UCN can additionally be assumed related to concrete's selfhealing capacity. Finer cements were found producing relatively high surface area density in ITZs. As a result, higher capacity of self-healing is expected for concretes made by fine cement type.

The mechanical part of the self-healing capacity deals with the direct interference of cracks and UCN. This is quantitatively evaluated, but will be discussed in a next paper. Further comparison with quantitative result in simulation and previous experiments will be conducted at that stage. One possible method considering interference of cracks and UCN is shown in Fig. 13. UCN missed by the crack can additionally 
provide a more delayed contribution by diffusion through the pores. Spacing of the UCN can be expected an important parameter under such conditions.

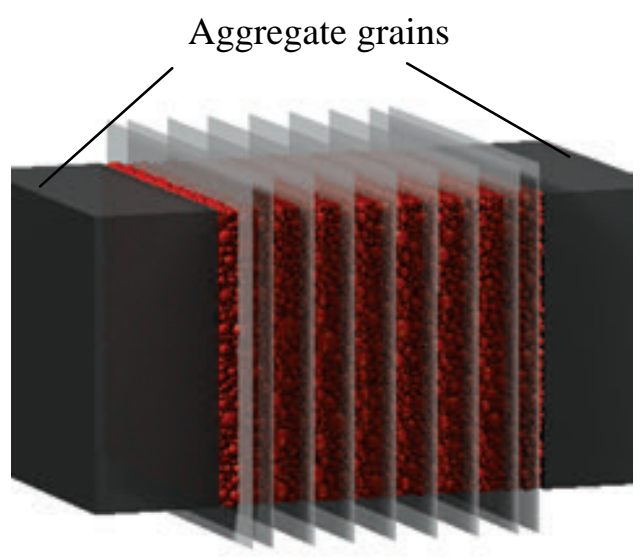

Series of cracks

Fig. 13. Evaluation of cracks interfering with UCN to provide gradient information on local self-healing capacity.

\section{CONCLUSIONS}

A mixture of composition and configurationsensitive descriptors of material structure can provide reliable quantitative information on the evolution process of unhydrated cement nuclei during maturation of a range of cement pastes. This can be achieved by concurrent algorithm-based computer simulated systems, like SPACE.

The water to cement ratio (w/c) is a key factor for self-healing capacity of concrete. For w/c $\geq 0.4$, the amount of UCN is at a negligible level. Hence, discussion on self-healing capacity of concrete is only sensible for a limited, but for HPC relevant range of $\mathrm{w} / \mathrm{c}$ ratios $(\mathrm{w} / \mathrm{c} \leq 0.4)$.

Concrete made with finer cement has high surface area density and equal volume fraction of UCN for given w/c ratio, so that relatively high probability of self-healing is expected for such types of concrete.

\section{REFERENCES}

Baldie KD (1985). Crack growth in hardened cement paste. PhD Thesis. Imperial College of Science and Technology. London, UK.

Chen H, Stroeven P, Stroeven M, Sluys LJ (2005). Nearest surface spacing between neighbouring aggregate particles in concrete: theoretical solution, In: Lakshmanan N, Gopalakrishnan S, Sreenath HG, eds. Proceeding of
International Conference on Advances in Concrete Composites and Structures, 2005 Jan 6-8; Chennai, India. Chennai: Allied Publishers Private Limited: 157-68.

Chen H, Stroeven P, Ye G, Stroeven M (2006). Influence of boundary conditions on pore percolation in model cement paste. Key Eng Mater 302-303:486-92.

Farahat AM, Kawakami M, Tanabe T (1995). Experimental observation on microstructural behavior of concrete. $\mathrm{J}$ Mat Civ Engr 7:87-95.

Granger S, Loukili A, Pijaudier-Cabot G, Chanvillard G (2005). Mechanical characterization of the self-healing effect of cracks in Ultra High Performance Concrete (UHPC), In: Banthia N, Uomoto T, Bentur A, Shah SP, eds. Proceedings of ConMat'05 and Mindess Symposium, 2005 Aug 22-24; Vancouver, Canada (on CD).

Hu J (2004). Porosity of concrete; morphological study of model concrete. PhD Thesis, Delft: Delft University Press.

Jacobsen S, Marchand J, Hornain H (1995). SEM observations of the micro-structure of frost deteriorated and self-healing concretes. Cem Concr Res 25: 1781-90.

Jensen OM, Hansen PF (2001). Water-entrained cementbased materials I. Experimental principals and theoretical background. Cem Concr Res 31:647-54.

Kasperkiewicz J, Stroeven P (1991). Observations on crack healing in concrete. In: Brandt AM, Marshall IH, eds. Brittle Matrix Composites 3. Warsaw: Elsevier Applied Science, 164-73.

Liu X-Y, Yao W, Zheng X-F, Wu J-P (2005). Experimental study on self-healing performance of concrete. J Build Mater 8:184-8 (in Chinese).

Lusche M (1972). Beitrag zum bruchmechanismus von auf druck beabspruchtem normal und leichtbeton mit geslossenem gefuge. Schriftreihe der Zementindustrie, Beton-Verlag (in German).

Neville AM (1995). Properties of concrete $\left(4^{\text {th }}\right.$ Edn). Harlow: Longman Group limited.

Pigeon M (1969). The process of crack initiation and propagation in concrete. PhD Thesis, London: Imperial College.

Scrivener KL, Crumbie AK, Laugesen P (2004). The interfacial transition zone (ITZ) between cement paste and aggregate in concrete. Interf Sci 12:411-21.

Stroeven M, Stroeven P (1997). Simulation of hydration and the formation of microstructure. In: Owen DRJ, Onate E, Hinton E, eds. Computational Plasticity. Barcelona: CIMNE, 981-7.

Stroeven M (1999). Discrete Numerical model for the structural assessment of composite materials. $\mathrm{PhD}$ Thesis, Delft: Delft University Press.

Stroeven M, Stroeven P (1999). SPACE system for simulation of aggregated matter application to cement hydration. Cem Concr Res 29:1299-304. 
Stroeven P (1973). Some aspects of the micromechanics of concrete. PhD Thesis, Delft: Delft University Press.

Struble LJ, Stutzman PE, Fuller JER (1989). Microstructural aspects of the fracture of hardened cement paste. J Am Ceram Soc 72: 2295-9.

Turner L (1937). The autogenous healing of cement and concrete: its relation to vibrated concrete and cracked concrete, Proceedings of International Association for Testing Materials. London: 344.

van Breugel K (1997). Simulation of hydration and formation of structure in hardening cement- based materials. Delft: Delft University Press. 